ANNALES

POLONICI MATHEMATICI

$88.2(2006)$

\title{
Graphs with multiple sheeted pluripolar hulls
}

\author{
by Evgeny Poletsky (Syracuse, NY) and \\ JAN WIEGERINCK (Amsterdam)
}

\begin{abstract}
We study the pluripolar hulls of analytic sets. In particular, we show that hulls of graphs of analytic functions can be multiple sheeted and sheets can be separated by a set of zero dimension.
\end{abstract}

1. Introduction. One of the oldest interesting topics in complex analysis is the problem of analytic extensions: find the maximal analytic object containing a given one. For example, if $f$ is an analytic function we are looking for its analytic continuation and if $A$ is an irreducible analytic set we try to find another one of the same dimension containing $A$.

The counterpart of an analytic extension in pluripotential theory is the so-called pluripolar hull. There are two types of pluripolar hulls of a set $A$ in a domain $D \subset \mathbb{C}^{n}$. Let $\operatorname{PSH}(D)$ be the set of all plurisubharmonic functions on $D$ and $\operatorname{PSH}_{0}(D)$ the set of all negative functions from $\operatorname{PSH}(D)$. Define

$$
\begin{aligned}
& A_{D}^{*}=\left\{z \in D:\left.\forall h \in \operatorname{PSH}(D) h\right|_{A}=-\infty \Rightarrow h(z)=-\infty\right\}, \\
& A_{D}^{-}=\left\{z \in D:\left.\forall h \in \operatorname{PSH}_{0}(D) h\right|_{A}=-\infty \Rightarrow h(z)=-\infty\right\} .
\end{aligned}
$$

For example, if $A$ is an analytic set in a pseudoconvex domain $D$, then every point of $A$ has a neighborhood $V$ where $A \cap V=\left\{h_{1}=\cdots=h_{k}=0\right\}$ and the functions $h_{k}$ are holomorphic on this neighborhood. Hence $A \cap V=$ $\left\{\log \max \left\{\left|h_{1}\right|, \ldots,\left|h_{k}\right|\right\}=-\infty\right\}$. In fact, $A_{D}^{*}=A$, because by [2, Cor. 1] there even exists $v \in \operatorname{PSH}(D)$ such that $A=\{v=-\infty\}$.

If such a $v \in \operatorname{PSH}(D)$ exists for $A$, we call $A$ pluricomplete in $D$. In general, an analytic extension of $A$ is contained in $A_{D}^{*}$.

In the case when $A=\Gamma_{f}$ is the graph of an analytic function $f$ it was conjectured in [9] that the closure of the analytic extension of $A$ coincides

2000 Mathematics Subject Classification: Primary 32U15; Secondary 32D15.

Key words and phrases: pluripotential theory, pluripolar hulls.

The first author was partially supported by an NSF grant. 
with $A_{D}^{*}$. However, A. Edigarian and the second author found in [3] an analytic function $f$ on the unit disk $\mathbb{D}$ that does not extend analytically while the pluripolar hull of its graph is the graph of an analytic function defined on almost the whole plane (cf. [11]). Thus pluripolar hulls and analytic extensions may differ by large sets.

The pluripolar hull of the graph $\Gamma_{f}$ of a holomorphic function $f(z)$ on a domain $D$ may well be multi-sheeted over $D$. The principal value of $\sqrt{z}$ on $\{\Re z>0\}$ provides the easiest example. Only recently did Zwonek [12], and, independently, Edlund and Jöricke [6], give examples of holomorphic functions $f$ on their domain of existence $D$ with the property that the pluripolar hull $\left(\Gamma_{f}\right)_{\mathbb{C}^{2}}^{*}$ is multi-sheeted over (parts of) $D$. In all these examples the topological codimension of $\partial D$ is 1 .

As we show in Section 2, this is an intrinsic property of analytic extensions: Let $E$ be a closed subset of $\mathbb{C}^{n+1}$ and $A$ be an analytic set of pure dimension $n$ in $\mathbb{C}^{n+1} \backslash E$. If the set $A_{\mathbb{C}^{n+1}}^{*}$ is analytic, then $\operatorname{dim} E \geq 2 n-1$. However, as we also show, there is a Cantor type set $K$ and a holomorphic function $f(z)$ on $D=\mathbb{C} \backslash K$ such that $\left(\Gamma_{f}\right)_{\mathbb{C}^{2}}^{*}$ is 2-sheeted over $D$. So for pluripolar extensions sheets can be separated by a 0-dimensional cut and this demonstrates another principal difference between analytic extensions and pluripolar hulls.

As a by-product we obtain an example of a uniformly convergent sequence of holomorphic functions such that their pluripolar hulls do not converge to the pluripolar hull of the limit.

The set $K$ should be sufficiently fat. Edigarian and the second author showed that if $D=\mathbb{C} \backslash K$, with $K$ a polar compact set in $\mathbb{C}$, and if $f$ is not extendible over $K$, then $\left(\Gamma_{f}\right)_{\mathbb{C}^{2}}^{*} \cap D \times \mathbb{C}=\Gamma_{f}$; see [4], and [5] for the fact that also over $K$ the hull is at most single sheeted.

2. Pluripolar extensions. Let $E$ be a closed set in a pseudoconvex domain $D \subset \mathbb{C}^{n}$. If $A \subset D \backslash E$ then, in general, $A_{D \backslash E}^{*}$ is a proper subset of $A_{D}^{*} \backslash E$. However, as the following statement shows, these sets coincide when $E$ is pluripolar.

Proposition 2.1. If $E$ is a closed pluripolar set in a pseudoconvex domain $D \subset \mathbb{C}^{n}$ and $A \subset D \backslash E$, then $A_{D}^{*} \backslash E=A_{D \backslash E}^{*}$.

Proof. Let $\left\{D_{j}\right\}$ be an increasing sequence of relatively compact subdomains with $\bigcup_{j} D_{j}=D$. By $\left[10\right.$, Thm. 2.4], $A_{D}^{*}=\bigcup_{j}\left(A \cap D_{j}\right)_{D_{j}}^{-}$. If $u \in \operatorname{PSH}_{0}\left(D_{j} \backslash E\right)$, then $u$ extends as a negative plurisubharmonic function to $D_{j}$ (see [8, Thm. 2.9.22]). Therefore, $\left(A \cap D_{j}\right)_{D_{j}}^{-} \backslash E=\left(A \cap D_{j}\right)_{D_{j} \backslash E}^{-}$. Since $\left(A \cap D_{j}\right)_{D_{j} \backslash E}^{-} \subset A_{D \backslash E}^{*}$, we see that $A_{D}^{*} \backslash E \subset A_{D \backslash E}^{*}$ and, consequently, $A_{D}^{*} \backslash E=A_{D \backslash E}^{*}$. 
The proposition below describes the situation when $E$ is a closed set in a pseudoconvex domain $D \subset \mathbb{C}^{n}, A \subset D \backslash E$ is an analytic set and $A_{D}^{*}$ is also analytic.

Proposition 2.2. Suppose that $E$ is a closed set in a pseudoconvex domain $D \subset \mathbb{C}^{n}$ and $A \subset D \backslash E$ is an analytic set. If the set $A_{D}^{*}$ is analytic then every irreducible component of $A_{D}^{*}$ contains a component of $A$ of the same dimension.

Proof. Let $X$ be an irreducible component of $A_{D}^{*}$. We represent $A_{D}^{*}$ as $X \cup Y$, where $Y$ is another analytic set in $D$ and $\operatorname{dim} X \cap Y<\operatorname{dim} X$. As indicated in Section 1, the set $Y$ is pluricomplete and $Y_{D}^{*}=Y$. It is easy to check that if $F, G \subset D$, then $(F \cup G)_{D}^{*}=F_{D}^{*} \cup G_{D}^{*}$. So if $B=A \cap(X \backslash Y)$ then $X \backslash Y \subset B_{D}^{*}$.

Suppose that $\operatorname{dim} X>\operatorname{dim} B$ and let $R$ be the set of regular points of $X$. The set of singular points of $X$ is analytic and, consequently, pluricomplete. By the argument above $X \backslash Y$ belongs to the pluripolar hull of the set $B^{\prime}=B \cap R$.

We may assume that $0 \in R$ and let $T$ be the tangent plane to $R$ at 0 . If $p$ is a projection of $\mathbb{C}^{n}$ on $T$, then the set $p\left(B^{\prime}\right)$ is pluripolar in $T$ and, consequently, there is a plurisubharmonic function $u$ on $T$ equal to $-\infty$ on $p\left(B^{\prime}\right)$. The set $p(R)$ has a non-empty interior in $T$ and, therefore, there is a point $z_{0} \in R$ such that $u\left(p\left(z_{0}\right)\right) \neq-\infty$. Then the function $v=u \circ p$ is plurisubharmonic on $\mathbb{C}^{n}$, equal to $-\infty$ on $B^{\prime}$ and $v\left(z_{0}\right)>-\infty$. Thus $R$ does not belong to the pluripolar hull of the set $B^{\prime}$. This contradiction proves the proposition.

Suppose that $A$ is a pluricomplete analytic set of pure dimension $m$ in $D \backslash E$. If $A_{D}^{*}$ is an analytic set in $D$ and $A$ is a proper subset of $A_{D}^{*} \backslash E$, then the set $E \cap A_{D}^{*}$ cuts $A_{D}^{*}$ into several pieces and, therefore, its topological dimension must be at least $2 m-1$.

For example, let $D=\left\{(z, w) \in \mathbb{C}^{2}\right\}$ and $E=\{\Im z=0, \Re z \geq 0\}$. Take a branch $w=f(z)$ of the function $w=\sqrt{z}$ over $\mathbb{C} \backslash E$ and let $A=$ $\{(z, f(z)): z \in \mathbb{C}\}$. The pluripolar hull of $A$ in $\mathbb{C}^{2} \backslash E$ is $A$ because the function $\log |f(z)-z|$ is equal to $-\infty$ exactly on $A$. But $A_{D}^{*}=\left\{(z, w): z=w^{2}\right\}$. In this example $A_{D}^{*}$ is an analytic set and the set $A_{D}^{*} \cap E$ is the real curve $\left\{\left(x^{2}, x\right): x \in \mathbb{R}\right\}$ which projects 2 to 1 except at 0 and its projection has dimension 1 .

As the following statement shows, this is the minimal possible dimension.

Proposition 2.3. Let $E$ be a closed set in a pseudoconvex domain $D \subset$ $\mathbb{C}^{n}$ and let $A$ be an irreducible analytic set of dimension $m$ in $D \backslash E$ such that $A_{D}^{*}$ is also analytic. If $p$ is a projection of $\mathbb{C}^{n}$ onto $\mathbb{C}^{m} \subset \mathbb{C}^{n}$ such that 
$p(A)$ has a non-empty interior in $\mathbb{C}^{m}$ and the topological dimension of $p(E)$ is less than $2 m-1$, then $A_{D}^{*} \backslash E=A$.

Proof. By Proposition 2.2 every irreducible component of $A_{D}^{*}$ contains a component of $A$ of the same dimension. Thus $A_{D}^{*}$ is also irreducible and has dimension $m$. We denote by $X$ the set of singular points of $A_{D}^{*}$. This set is analytic and its dimension is at most $m-1$. Hence the set $Y=A_{D}^{*} \backslash X$ is an irreducible complex submanifold of $D \backslash X$. Let $X^{\prime} \subset Y$ be the set where the restriction of the projection $p$ to $Y$ has rank less than $m$. Since $p(A)$ has non-empty interior in $\mathbb{C}^{m}$, the set $Y^{\prime}=Y \backslash X^{\prime}$ is non-empty and relatively open in $Y$. The set $X^{\prime}$ is analytic and, therefore, its dimension is at most $m-1$. Hence the set $Y^{\prime}$ is an irreducible complex submanifold of $D \backslash\left(X \cup X^{\prime}\right)$ and the set $A^{\prime}=A \cap Y^{\prime}$ is a non-empty submanifold of $Y^{\prime} \backslash E$ of dimension $m$.

Suppose that the topological dimension of $p(E)$ is at most $2 m-2$. The projection $p$ is locally homeomorphic on $Y^{\prime}$ and, therefore, the set $E \cap Y^{\prime}$ also has dimension at most $2 m-2$. Since $E$ is closed the set $A^{\prime}$ is relatively open in $Y^{\prime}$. The set $Y^{\prime \prime}=Y^{\prime} \backslash\left(A^{\prime} \cup E\right)$ is also relatively open in $Y^{\prime}$. Thus $Y^{\prime}$ is a topological manifold of dimension $2 m$ and equals the union of two open sets $A^{\prime}$ and $Y^{\prime \prime}$ and a closed set $E$ of dimension at most $2 m-2$. By the Urysohn-Menger Theorem a set of dimension $2 m-2$ cannot disconnect a $2 m$-dimensional manifold (see [7, Ch. IV.5, Cor. 1]). Hence either $A^{\prime}$ or $Y^{\prime \prime}$ is empty. Since $A^{\prime}$ is not empty, $Y^{\prime} \backslash E=A^{\prime}$.

Since $A$ is closed in $A_{D}^{*} \backslash E$, it contains $X \backslash E$ and $X^{\prime} \backslash E$. So $A_{D}^{*} \backslash E=A$.

When $A$ is an analytic set in $D \backslash E$ we denote by $A_{E}$ the intersection of $E$ and the closure of $A$ in $D$. If $A_{D}^{*} \backslash E \neq A$, we will say that $A$ has a non-trivial pluripolar extension through $E$ in $D$.

The following theorem lists some limitations on the set $A_{E}$ when a nontrivial pluripolar extension takes place. Following [1] we call a set $G$ in a domain $Y \subset \mathbb{C}^{p}$ locally removable if $G$ is closed and for every open set $V$ in $Y$ every bounded holomorphic function $f$ on $V \backslash G$ extends holomorphically to $V$.

Theorem 2.4. Suppose that $D$ is a pseudoconvex domain in $\mathbb{C}^{n}, E$ is a closed set in $D$ and $A$ is an analytic set of pure dimension $m$ in $D \backslash E$ with a non-trivial pluripolar extension through $E$ in $D$. Then the $(2 m-1)$-Hausdorff measure of the set $A_{E}$ is not equal to zero and if, additionally, $p: \mathbb{C}^{n} \rightarrow \mathbb{C}^{m}$ is a projection such that the restriction $\left.p\right|_{A_{E}}$ is proper and $A \cap p^{-1}(z)$ is empty for all $z \in p\left(A_{E}\right)$, then $p\left(A_{E}\right)$ is not locally removable in $\mathbb{C}^{m}$.

Proof. The set $A$ is analytic in $D \backslash A_{E}$. If the (2m-1)-Hausdorff measure of $E$ is zero, then by Shiffman's theorem (see [1, 4.4]) the closure $\bar{A}$ of $A$ in $D$ is an analytic set in $D$. Since the domain $D$ is pseudoconvex there is 
a holomorphic function $f$ on $D$ such that $\bar{A}=\{f=0\}$. Thus $A_{D}^{*}=\bar{A}$ and this contradicts the assumption that the extension is non-trivial.

In the second case, if $p\left(A_{E}\right)$ is locally removable in $\mathbb{C}^{m}$, then by the Proposition in $[1,18.1]$ the closure $\bar{A}$ of $A$ is an analytic set in $D$ as before and the same argument leads to a contradiction.

In our main example, $n=2, m=1$. In this case Theorem 2.4 can be reformulated as follows:

COROllary 2.5. If in the assumptions of Theorem 2.4, $n=2$ and $m=1$, then the first Hausdorff measure of $A_{E}$ is not zero and, under additional assumptions, the first Hausdorff measure of $p\left(A_{E}\right)$ is not zero.

\section{A holomorphic function on the complement of a Cantor type set with 2-sheeted hull}

Definition 3.1. A Cantor type set $K$ is a compact perfect subset of $\mathbb{R}$ with empty interior.

It is a well known fact from elementary point set topology that such a $K$ is homeomorphic to Cantor's middle thirds set. It is of the form $\left[a_{0}, b_{0}\right] \backslash$ $\bigcup_{j=1}^{\infty} I_{j}$ where $I_{j}$ are open intervals in $\left[a_{0}, b_{0}\right], \bar{I}_{j} \cap \bar{I}_{k}=\emptyset$ if $j \neq k$ and $\bigcup_{j=1}^{\infty} I_{j}$ is dense in $[a, b]$. We can assume that the length of $I_{j}=\left(a_{j}, b_{j}\right)$ decreases with $j$.

It is useful to enumerate the set $\left\{a_{j}, b_{j}: j=0, \ldots, n\right\}$ as $\left\{\alpha_{j n}, \beta_{j n}\right\}$ so that $\alpha_{0 n}=a_{0}, \alpha_{j n}<\beta_{j n}<\alpha_{j+1, n}$ and $\beta_{n n}=b_{0}$. Note that $\left[a_{0}, b_{0}\right] \backslash$ $\bigcup_{j=1}^{n} I_{j}=\bigcup_{j=0}^{n}\left[\alpha_{j n}, \beta_{j n}\right]$ and that $I_{m} \cap\left[\alpha_{j n}, \beta_{j n}\right] \neq \emptyset$ implies $I_{m} \subset$ $\left[\alpha_{j n}, \beta_{j n}\right] \neq \emptyset$.

Let

$$
g_{n}(z)=\frac{z-a_{0}}{z-b_{0}} \frac{z-b_{1}}{z-a_{1}} \cdots \frac{z-b_{n}}{z-a_{n}}
$$

Then

$$
g_{n}(z)=\frac{z-\alpha_{0 n}}{z-\beta_{0 n}} \frac{z-\alpha_{1 n}}{z-\beta_{1 n}} \cdots \frac{z-\alpha_{n n}}{z-\beta_{n n}} .
$$

Each fraction $\frac{z-\alpha_{j n}}{z-\beta_{j n}}, j=0,1, \ldots, n$, has a holomorphic branch $\sqrt{\frac{z-\alpha_{j n}}{z-\beta_{j n}}}$ of its square root outside $\left[\alpha_{j n}, \beta_{j n}\right]$ that equals 1 at infinity. Let

$$
f_{n}(z)=\sqrt{\frac{z-\alpha_{0 n}}{z-\beta_{0 n}}} \sqrt{\frac{z-\alpha_{1 n}}{z-\beta_{1 n}}} \cdots \sqrt{\frac{z-\alpha_{n n}}{z-\beta_{n n}}}=\sqrt{g_{n}(z)} .
$$

Then $f_{n}(\infty)=1, f_{n}^{2}=g_{n}$ and $f_{n}$ is holomorphic on $G_{n}=\left(\mathbb{C} \backslash\left[a_{0}, b_{0}\right]\right) \cup$ $\bigcup_{j=1}^{n} I_{j}$.

The maximal analytic extension of $f_{n}$ is a branched 2-sheeted cover $X_{n}=$ $\left\{(z, w): w^{2}=g_{n}(z)\right\}$ of $\mathbb{C}$ that branches over $\left\{a_{j}, b_{j}: j=0,1, \ldots, n\right\}$. The pluripolar hull $\left(\Gamma_{f_{n}}\right)_{\mathbb{C}^{2}}^{*}$ equals $X_{n}$. 
LEMma 3.2. Keeping the above notation, the sequence $\left\{g_{n}\right\}$ converges normally to an analytic function $g$ on $\mathbb{C} \backslash K$. Moreover, the function $g$ extends analytically over a point $x \in K$ if and only if for some $\alpha<x<\beta$ the length of the set $K \cap(\alpha, \beta)$ is zero.

Proof. Let $L$ be a compact set in $\mathbb{C} \backslash K$. Let us show that

$$
\frac{z-a_{0}}{z-b_{0}} \prod_{j=1}^{\infty} \frac{z-b_{j}}{z-a_{j}}=\lim _{n \rightarrow \infty} g_{n}(z)
$$

is uniformly convergent on $L$. There exists $n_{0}$ such that $L \subset G_{n}$ for $n>n_{0}$ and moreover, for some $\delta>0$,

$$
L \subset\left\{z:\left|z-a_{j}\right|>\delta, j=0,1, \ldots\right\} .
$$

Hence, for $z \in L$,

$$
\left|\frac{z-b_{j}}{z-a_{j}}-1\right|=\left|\frac{b_{j}-a_{j}}{z-a_{j}}\right| \leq \frac{b_{j}-a_{j}}{\delta} .
$$

Since $\sum\left(b_{j}-a_{j}\right)$ is finite, the product in (3.2) converges uniformly on $L$ to a function $g$ that is holomorphic on $\mathbb{C} \backslash K$.

Suppose that $g$ extends analytically over a point $x \in K$ so that $g$ is analytic on $(\mathbb{C} \backslash K) \cup(\alpha, \beta)$. We may assume that $\alpha \in I_{k}, b_{k} \leq x$, and $\beta \in I_{m}, a_{m} \geq x$, and

$$
g_{1 n}(z)=\prod \frac{z-\alpha_{j n}}{z-\beta_{j n}},
$$

where the product runs over all $j$ such that either $\beta_{j n}<\alpha$ or $\alpha_{j n}>\beta$. Let

$$
g_{2 n}(z)=\prod \frac{z-\alpha_{j n}}{z-\beta_{j n}}
$$

where the product runs over all $j$ such that $\alpha<\alpha_{j n}$ and $\beta_{j n}<\beta$. Then $g_{n}=g_{1 n} g_{2 n}$ and by the argument above the sequences $\left\{g_{1 n}\right\}$ and $\left\{g_{2 n}\right\}$ converge uniformly on compacta in $\mathbb{C} \backslash(K \backslash(\alpha, \beta))$ and $\mathbb{C} \backslash(K \cap(\alpha, \beta))$ respectively. We denote their respective limits by $g_{1}$ and $g_{2}$.

For the derivative of $g_{2 n}$ we find $g_{2 n}^{\prime}(\infty)=\sum\left(\beta_{j n}-\alpha_{j n}\right)=l_{n}$, where the sum runs over all $j$ such that $\alpha<\alpha_{j n}$ and $\beta_{j n}<\beta$. Thus $g_{2 n}(\infty)$ is equal to the length $l_{n}$ of the intervals $\left(\alpha_{j n}, \beta_{j n}\right)$ lying in $(\alpha, \beta)$ and $g_{2}^{\prime}(\infty)$ is the length of the set $K \cap(\alpha, \beta)$. If this length is positive, then the function $g_{2}$ is not constant and, therefore, does not extend to $K \cap(\alpha, \beta)$.

If this length is 0 then for $z \in \mathbb{C}$ such that $|z-y| \geq 1$ for all $y \in(\alpha, \beta)$ we have

$$
\left|1-g_{2 n}(z)\right|=\left|1-\prod\left(1+\frac{\beta_{j n}-\alpha_{j n}}{z-\beta_{j n}}\right)\right| \leq e^{l_{n}}-1 .
$$

Hence the sequence $\left\{g_{2 n}\right\}$ converges to 1 near $\infty, g_{2} \equiv 1$ and $g$ extends analytically over $(\alpha, \beta)$. 
LEMMA 3.3. If $f=f_{K}$ and the length of $K$ is positive, then the union $\bar{\Gamma}_{f} \cup \bar{\Gamma}_{-f}$ of the closures of the graphs of $f$ and $-f$ is not an analytic set.

Proof. If $A=\bar{\Gamma}_{f} \cup \bar{\Gamma}_{-f}$ is an analytic set, then there is a holomorphic function $h=h(z, w)$ on $\mathbb{C}^{2}$ such that $h \equiv 0$ on $A$. We have $A=\Gamma_{f} \cup \Gamma_{-f} \cup E$, where $E \subset K \times \mathbb{C}$.

Let us show that for every $z_{0} \in K$ the analytic set $E_{z_{0}}=\left\{w:\left(z_{0}, w\right) \in E\right\}$ $=A \cap\left\{z_{0}\right\} \times \mathbb{C}$ consists of at most two points. If it contains three points, then at least two of them belong to, say, $\bar{\Gamma}_{f}$. Thus there are sequences $\left\{z_{j}\right\}$ and $\left\{z_{j}^{\prime}\right\}$ converging to $z_{0}$ such that the sequences $\left\{f\left(z_{j}\right)\right\}$ and $\left\{f\left(z_{j}^{\prime}\right)\right\}$ have distinct limits. Connecting each $z_{j}$ and $z_{j}^{\prime}$ by small curves in $\mathbb{C} \backslash K$ and looking at their limits we see that the cluster set of $f$ at $z_{0}$ contains a continuum. Hence, $E_{z_{0}}=\mathbb{C}$ and $h\left(z_{0}, w\right) \equiv 0$.

From the Taylor expansion of $h$ we immediately derive that $h(z, w)=$ $\left(z-z_{0}\right)^{n} h_{1}\left(z_{0}, w\right)$, where $h_{1}$ is holomorphic on $\mathbb{C}^{2}$ and $h_{1}\left(z_{0}, w\right) \not \equiv 0$. But for every point $w \in \mathbb{C}$ there is a sequence of $z_{j}$ converging to $z_{0}$ such that, say, $f\left(z_{j}\right)$ converges to $w$. Since $h\left(z_{j}, f\left(z_{j}\right)\right)=0$ we see that $h_{1}\left(z_{0}, w\right)=0$. This contradiction shows that $E_{z_{0}}$ has at most two points and the intersection of $\bar{\Gamma}_{f}$ or $\bar{\Gamma}_{-f}$ with $E$ consists of at most one point.

It follows that $f$ extends continuously to $K$. Since $K$ lies on the real line, $f$ extends holomorphically to $\mathbb{C}$; but this impossible by Lemma 3.2.

EXAMPLE 3.4. If the set $K$ has Lebesgue measure 0, then

$$
\lim _{n \rightarrow \infty} g_{n}(z)=1
$$

uniformly on any compact set $L$ not meeting $K$. It follows that $f \equiv 1$ and $\left(\Gamma_{f}\right)_{\mathbb{C}^{2}}^{*}=\{(z, 1)\}$. But the Hausdorff limit of the sets $X_{n}$ over $D$ equals $\{(z, w): w=1$ or -1$\}$.

We will need the next lemma, whose proof is similar to the proof of Theorem 2.1 in [4].

LEMMA 3.5. Let $f$ be a holomorphic function on a domain $V \subset \mathbb{C}^{N}$ containing a closed ball $B$ and let $\left\{r_{n}\right\}_{n}$ be a sequence of rational functions of degree $n$ with poles outside $V$ and such that the sup-norm $\left\|f-r_{n}\right\|_{B}^{1 / n}$ tends to 0 as $n \rightarrow \infty$. Then there is a plurisubharmonic function $v$ on $\mathbb{C}^{N+1}$ such that $\{v=-\infty\} \cap(V \times \mathbb{C})=\Gamma_{f}$. Thus, $\left(\Gamma_{f}\right)_{\mathbb{C}^{N+1}}^{*} \cap(V \times \mathbb{C})=\Gamma_{f}$.

Proof. The functions $r_{n}$ are ratios of polynomials $p_{n}$ and $q_{n}$ of degree $n$. We may assume that $B$ is the closed unit ball centered at the origin and $\left\|q_{n}\right\|_{B}=1$. Then $\left|q_{n}(z)\right| \leq \max \left\{1,|z|^{n}\right\},\left\|p_{n}\right\|_{B}$ does not exceed some constant $C$ and $\left|p_{n}(z)\right| \leq C \max \left\{1,|z|^{n}\right\}$.

Consider the plurisubharmonic functions

$$
u_{n}(z, w)=\frac{1}{n} \log \left|q_{n}(z) w-p_{n}(z)\right|
$$


on $\mathbb{C}^{N+1}$. From the estimates on $p_{n}$ and $q_{n}$ there is a constant $C_{1}$ such that $u_{n}(z, w) \leq 2 \log |z|+\log |w|+C_{1}$ when $|z| \geq 1$ and $u_{n}(z, w) \leq \log |w|+C_{1}$ when $|z| \leq 1$.

We take $z_{n} \in B$ such that $q_{n}\left(z_{n}\right)=a_{n},\left|a_{n}\right|=1$. Let $w_{n}=\left(p_{n}\left(z_{n}\right)+1\right) / a_{n}$. Then $\left|w_{n}\right| \leq C+1$ and $u_{n}\left(z_{n}, w_{n}\right)=0$. If $B_{n}$ is a ball in $\mathbb{C}^{N+1}$ centered at $\left(z_{n}, w_{n}\right)$ and of radius $r_{n}=C+5$, then $B_{n}$ contains the unit ball $B^{\prime}$ centered at the origin and

$$
\int_{B_{n}} u_{n} d V \geq c u_{n}\left(z_{n}, w_{n}\right)=0
$$

It is immediate from the upper estimates on $u_{n}$ that there is a constant $C_{2}$ such that

$$
\int_{B^{\prime}} u_{n} d V \geq C_{2}
$$

By our assumption there is a sequence $\left\{d_{n}\right\}$ converging to $\infty$ such that

$$
u_{n}\left(z, f_{n}(z)\right)=\frac{1}{n} \log \left|q_{n}(z)\right|+\frac{1}{n} \log \left|f(z)-\frac{p_{n}(z)}{q_{n}(z)}\right| \leq-d_{n}
$$

when $|z| \leq 1$.

Let us take a sequence $\left\{c_{n}\right\}$ of positive reals such that $\sum c_{n}=1$ while $\sum c_{n} d_{n}=\infty$. Let

$$
v(z, w)=\sum_{n=1}^{\infty} c_{n} \max \left\{u_{n}(z, w), d_{n}\right\}
$$

Since

$$
\int_{B} v d V \geq C_{2}
$$

we see that $v \not \equiv-\infty$ and, therefore, it is a plurisubharmonic function on $\mathbb{C}^{N+1}$. Clearly, $v(z, f(z))=-\infty$ when $|z| \leq 1$. Therefore, $v=-\infty$ on $\Gamma_{f}$.

The zeros of the polynomials $q_{n}$ are in $\mathbb{C}^{N} \backslash V$. Hence the functions $h_{n}(z)=n^{-1} \log \left|q_{n}(z)\right|$ are harmonic on $V$ and uniformly bounded above on compacta. So if $z \in V$ and $\liminf h_{n}(z)=-\infty$, then there is a subsequence $\left\{n_{k}\right\}$ such that $\lim h_{n_{k}}(z)=-\infty$. Therefore, the functions $h_{n_{k}}$ converge to $-\infty$ uniformly on compacta in $V$. But $h_{n_{k}}\left(z_{n_{k}}\right)=0$, and this contradiction tells us that $\lim \inf h_{n}(z)>-\infty$. So if $w \neq f(z)$, then

$$
v(z, w) \geq \sum_{n=1}^{\infty} c_{n} h_{n}(z)+\sum_{n=1}^{\infty} \frac{c_{n}}{n} \log \left|w-\frac{p_{n}(z)}{q_{n}(z)}\right|>-\infty .
$$

Hence $\{v=-\infty\} \cap(V \times \mathbb{C})=\Gamma_{f}$ and $\left(\Gamma_{f}\right)_{\mathbb{C}^{N+1}}^{*} \cap(V \times \mathbb{C})=\Gamma_{f}$.

Now we can present our main example.

Theorem 3.6. There exists a Cantor type set $K$, obtained by deleting intervals $I_{i}=\left(a_{i}, b_{i}\right)$ from $[-1,1]$, such that the function $f=f_{K}$ given by 
Lemma 3.3 has the following property:

$$
\left(\Gamma_{f}\right)_{\mathbb{C}^{2}}^{*} \cap(\mathbb{C} \backslash K) \times \mathbb{C}=\Gamma_{f} \cup \Gamma_{-f}
$$

Proof. We will construct $K$ by deleting a sequence of open intervals $\left(a_{i}, b_{i}\right)$ from the interval $[-1,1]$. For convenience, set $a_{0}=1, b_{0}=-1$. In order to choose the intervals appropriately, we have to construct certain subdomains $D_{n}$ in the open unit disk $\mathbb{D}$ in the process. The domains $D_{n}$ will contain the set $\mathbb{D} \cap\{|\Im z|>1 / 2\}$. Thus the closed discs $S=\{|z+3 i / 4| \leq 1 / 8\}$ and $X=\{|z-3 i / 4| \leq 1 / 8\}$ will be contained in $D_{n}$.

For a compact set $F$ in a domain $D \subset \mathbb{C}$ let

$$
\omega(z, F, D)=-\sup \left\{h(z): h \in \mathrm{PSH}_{0}(D), \limsup _{w \rightarrow K} h(w) \leq-1\right\}
$$

be the harmonic measure of $F$ in $D$. We set $D_{0}=\mathbb{D}$ and observe that $\omega\left(z, S, D_{0}\right)>c_{0}$ for some positive $c_{0}$.

Let $\left\{c_{n}\right\}$ be a sequence of positive real numbers converging to $\infty$. Suppose that the intervals $I_{1}, \ldots, I_{n}$ have been chosen. We take as $a_{n+1}$ the midpoint of the largest interval in their complement. Next take $b_{n+1}>a_{n+1}$ so small that the interval $\left[a_{n+1}, b_{n+1}\right]$ does not intersect the intervals $I_{1}, \ldots, I_{n}$, $d_{n+1}=b_{n+1}-a_{n+1}<4^{-(n+1) c_{n+1}}$ and, moreover,

$$
\omega\left(z, S, D_{n+1}\right)>c_{0}, \quad z \in X .
$$

Here we define $D_{n+1}=D_{n} \backslash \mathbb{D}\left(a_{n+1},\left(b_{n+1}-a_{n+1}\right) 2^{n+1}\right)$, where $\mathbb{D}(a, r)$ is the open disk centered at $a$ and of radius $r$.

Observe that for $j \leq n$,

$$
\left|\frac{z-b_{j}}{z-a_{j}}-1\right|=\left|\frac{d_{j}}{z-a_{j}}\right|<\frac{1}{2^{j}}
$$

on $D_{n}$. It follows that

$$
\prod_{j=1}^{n} \frac{z-b_{j}}{z-a_{j}}
$$

is bounded on $D_{n}$ independently of $n$.

Let $z_{0} \in X$. We will show that $\left(z_{0},-f\left(z_{0}\right)\right) \in\left(\Gamma_{f}\right)_{\mathbb{C}^{2}}^{*}$. Then $\Gamma_{-f}$ is also in the hull and we are done. Consider the function $\widetilde{f}_{n}$ defined on $\mathbb{D} \backslash \bigcup_{j=1}^{n} I_{j}$ by

$$
\widetilde{f}_{n}(z)= \begin{cases}f_{n}(z) & \text { if } \Im z<0, \\ -f_{n}(z) & \text { if } \Im z>0, \\ \lim _{y \uparrow 0} f(x+i y) & \text { if } x \in[-1,1] \backslash \bigcup I_{j} .\end{cases}
$$

The function $\widetilde{f}_{n}$ is holomorphic. Let $c_{n}=\widetilde{f}_{n}\left(z_{0}\right)+f_{K}\left(z_{0}\right)$. Then $c_{n} \rightarrow 0$ as $n \rightarrow \infty$. The functions $h_{n}=\widetilde{f}_{n}-c_{n}$ tend to $f_{K}$ uniformly on compact sets in $\mathbb{D} \cap\{\Im z<0\}$ and $h_{n}\left(z_{0}\right)=-f_{K}\left(z_{0}\right)$. 
Now let $u$ be a plurisubharmonic function on $\mathbb{C}^{2}$ that equals $-\infty$ on $\Gamma_{f}$. The function $u\left(z, h_{n}(z)\right)$ is subharmonic on the domain $D_{n}$, and because $h_{n}(z)$ is bounded on $D_{n}$ independently of $n, u\left(z, h_{n}(z)\right)$ is bounded by a constant $M$ independently of $n$.

Next we apply the two constants theorem and find

$$
\begin{aligned}
u\left(z_{0},-f_{K}\left(z_{0}\right)\right) \leq & M\left(1-\omega\left(z_{0}, S, D_{n}\right)\right. \\
& +\max _{z \in S} u\left(z, h_{n}(z)\right) \omega\left(z_{0}, S, D_{n}\right) \rightarrow-\infty \quad \text { as } n \rightarrow \infty .
\end{aligned}
$$

Hence, $\left(\Gamma_{f}\right)_{\mathbb{C}^{2}}^{*} \supset \Gamma_{f} \cup \Gamma_{-f}$.

To get the equality we will show that the sup-norm satisfies

$$
\left\|g-g_{n}\right\|_{L}^{1 / n} \rightarrow 0, \quad n \rightarrow \infty,
$$

on compacta $L$ outside $K$. For this we write

$$
\left|g-g_{n}\right|=\left|g_{n}\right|\left|\prod_{k=n+1}^{\infty} \frac{z-a_{k}}{z-b_{k}}-1\right| \text {. }
$$

The first factor is bounded by a constant $C$ depending on $L$. To estimate the second factor we let $\delta$ be the distance from $L$ to $K$ and write the factor as

$$
\left|\prod_{k=n+1}^{\infty}\left(1+\frac{d_{k}}{z-b_{k}}\right)-1\right| \leq \prod_{k=n+1}^{\infty}\left(1+\frac{d_{k}}{\delta}\right)-1 \leq \exp \left(\sum_{k=n+1}^{\infty} \frac{d_{k}}{\delta}\right)-1 .
$$

Since $d_{k}<4^{-k c_{k}}$ we see that

$$
\left|\prod_{k=n+1}^{\infty} \frac{z-a_{k}}{z-b_{k}}-1\right| \leq \exp \left(\frac{4^{-n c_{n}}}{\left(1-4^{-c_{n}}\right) \delta}\right)-1 .
$$

Hence

$$
\left\|g-g_{n}\right\|_{L}^{1 / n} \leq 2\left(\frac{C_{L}}{\delta}\right)^{1 / n} 4^{-c_{n}}
$$

when $n$ is sufficiently large and

$$
\left\|g-g_{n}\right\|_{L}^{1 / n} \rightarrow 0, \quad n \rightarrow \infty .
$$

By Lemma 3.5, $\Gamma_{g}^{*}=\Gamma_{g}$ in $\mathbb{C}^{2} \backslash(K \times \mathbb{C})$. Thus for any $\left(z_{0}, w_{0}\right)$ with $z_{0} \in$ $\mathbb{C} \backslash K$ and $w_{0} \neq g\left(z_{0}\right)$, there is a function $u \in \operatorname{PSH}\left(\mathbb{C}^{2}\right)$ such that $\left.u\right|_{\Gamma_{g}}=-\infty$ and $u\left(z_{0}, w_{0}\right) \neq-\infty$. Then the function $v(z, w)=u\left(z, w^{2}\right)$ is equal to $-\infty$ on $\Gamma_{f} \cup \Gamma_{-f}$ and $v\left(z_{0}, \pm \sqrt{w_{0}}\right) \neq-\infty$. Hence $\left(\Gamma_{f}\right)_{\mathbb{C}^{2}}^{*} \cap(\mathbb{C} \backslash K) \times \mathbb{C}=\Gamma_{f} \cup \Gamma_{-f}$.

\section{References}

[1] E. M. Chirka, Complex Analytic Sets, Math. Appl. (Soviet Ser.) 46, Kluwer, Dordrecht, 1989.

[2] M. Colţoiu, Complete locally pluripolar sets, J. Reine Angew. Math. 412 (1990), 108-112. 
[3] A. Edigarian and J. Wiegerinck, Graphs that are not complete pluripolar, Proc. Amer. Math. Soc. 131 (2003), 2459-2465.

[4] -, - , The pluripolar hull of the graph of a holomorphic function with polar singularities, Indiana Univ. Math. J. 52 (2003), 1663-1680.

[5] - - - Determination of the pluripolar hull of graphs of certain holomorphic functions, Ann. Inst. Fourier (Grenoble) 54 (2004), 2085-2104.

[6] T. Edlund and B. Jöricke, The pluripolar hull of a graph and fine analytic continuation, Ark. Mat., to appear; ArXiv:math.CV/0405025.

[7] W. Hurewicz and H. Wallman, Dimension Theory, Princeton Math. Ser. 4, Princeton Univ. Press, Princeton, 1941.

[8] M. Klimek, Pluripotential Theory, London Math. Soc. Monogr. 6, Clarendon Press, 1991.

[9] N. Levenberg, G. Martin and E. A. Poletsky, Analytic disks and pluripolar sets, Indiana Univ. Math. J. 41 (1992), 515-532.

[10] N. Levenberg and E. A. Poletsky, Pluripolar hulls, Michigan Math. J. 46 (1999), 151-162.

[11] J. Siciak, Pluripolar sets and pseudocontinuation, in: Complex Analysis and Dynamical Systems II (Nahariya, 2003), Contemp. Math. 382, Amer. Math. Soc., Providence, RI, 2005, 385-394.

[12] W. Zwonek, A note on pluripolar hulls of graphs of Blaschke products, Potential Anal. 22 (2005), 195-206.

Department of Mathematics

215 Carnegie Hall

Syracuse University

Syracuse, NY 13244, U.S.A.

E-mail: eapolets@syr.edu
Korteweg-de Vries Institute for Mathematics

University of Amsterdam

Plantage Muidergracht 24

1018 TV Amsterdam, The Netherlands

E-mail: janwieg@science.uva.nl 